\title{
Incontinência urinária: a atuação do profissional de enfermagem
}

\author{
Urinary incontinence: the performance of the nursing professional
}

Incontinencia urinaria: la actuación del profesional de enfermería

\author{
Layla Guimarães Paixão Oliveira ${ }^{1 *}$, Anderson Guimarães de Oliveira ${ }^{2}$, Gilberto de Souza ${ }^{1}$, Marcio \\ Antonio Resende'.
}

\section{RESUMO}

Objetivo: Compreender os aspectos fisiológicos e terapêuticos da incontinência urinária e sua influência na qualidade de vida do incontinente, apresentando propostas de como a enfermagem pode atuar para o bem-estar físico, emocional e social dos portadores. Métodos: Foram utilizadas como fonte de pesquisa as bases de dados científicos eletrônicos PubMed, SciELO, portal de periódicos CAPES, EBSCO e DynaMed e selecionados 32 artigos com recorte temporal de 2002 a 2018. Resultados e Discussão: Os enfermeiros podem prestar assistência aos incontinentes, sendo capazes para avaliar, identificar, fornecer informações e estabelecer algumas intervenções adequadas. Esses podem atuar com os portadores de incontinência, através da educação em saúde, do suporte emocional, da terapia comportamental e do processo de enfermagem. Considerações Finais: Poucos profissionais de enfermagem conhecem ou investigam a respeito desse tema, que necessita ser mais explorado e difundido, para o manejo adequado dos incontinentes. Os enfermeiros podem desempenhar um papel importante na identificação dos sintomas, interação com o paciente, incentivando mudanças comportamentais e o tratamento clínico.

Palavras-chave: Incontinência Urinária, Enfermagem, Qualidade de vida.

\section{ABSTRACT}

Objective: Understanding the physiological and therapeutic aspects of urinary incontinence and its influence on incontinent quality of life, presenting proposals on how nursing can act for the physical, emotional and social well-being of the patients. Methods: The electronic scientific databases PubMed, SciELO, portal of journals CAPES, EBSCO and DynaMed were selected and 32 articles with temporal cut from 2002 to 2018 were used. Results and Discussion: Nurses can provide assistance to incontinent, being able to evaluate, identify, provide information and establish some appropriate interventions. These can work with those with incontinence, through health education, emotional support, behavioral therapy and the nursing process. Final Considerations: Few nursing professionals know about or investigate this issue, which needs to be further explored and disseminated, for the proper management of incontinents. Nurses can have an performance role in identifying the symptoms, interacting with the patient, encouraging behavioral changes and clinical treatment.

Key words: Urinary incontinence, Nursing, Quality of life.

\section{RESUMEN}

Objetivo: Comprender los aspectos fisiológicos y terapéuticos de la incontinencia urinaria y su influencia en la calidad de vida del incontinente, presentando propuestas de cómo la enfermería puede actuar para el bienestar físico, emocional y social de los portadores. Métodos: Se utilizaron como fuente de investigación las bases de datos científicos electrónicos PubMed, SciELO, portal de revistas CAPES, EBSCO y DynaMed y seleccionados 32 artículos con recorte temporal de 2002 a 2018. Resultados y Discusion: Los enfermeros pueden prestar asistencia a los incontinentes, siendo capaces de evaluar, identificar, proporcionar información y establecer algunas intervenciones adecuadas. Estos pueden actuar con los portadores de incontinencia, a través de la educación en salud, del soporte emocional, de la terapia conductual y del proceso de enfermería. Consideraciones finales: Pocos profesionales de enfermería conocen o investigan acerca de ese tema, que necesita ser más explotado y difundido, para el manejo adecuado de los incontinentes. Los enfermeros pueden desempeñar un papel importante en la identificación de los síntomas, la interacción con el paciente, incentivando cambios conductuales y el tratamiento clínico.

Palabras clave: Incontinencia Urinaria, Enfermería, Calidad de vida.

${ }^{1}$ Centro Universitário Presidente Tancredo de Almeida Neves (UNIPTAN).

2 Escola Preparatória de Cadetes do Ar (EPCAR). *E-mail: laylagoliveira@hotmail.com 


\section{INTRODUÇÃO}

Incontinência urinária (IU) é definida como a perda involuntária de urina, que de acordo com a International Continence Society (ICS - Sociedade Internacional de Continência), gera um sério problema social e de higiene para o incontinente. Para o ser humano, apresentar incontinência é uma condição angustiante e de incapacidade, que afeta diretamente a vida em seus aspectos físicos, sociais, psicológicos, ocupacionais, domésticos e sexuais. Portanto, é um problema de saúde com dimensões mundiais e de grande impacto econômico, tanto para o sistema de saúde público quanto para o incontinente (CÂNDIDO et al., 2017; BENÍCIO et al., 2017; BORBA et al., 2008; ABRAMS et al.,2003).

Os portadores dessa patologia beneficiam-se quando expostos a tratamento específico, através de intervenções médicas e de enfermagem. No entanto, muitas pessoas não sabem disso, pois a IU é uma disfunção que não apresenta uma divulgação na área da saúde e nos meios de comunicação, deixando então de ser identificada e esclarecida para a população (OLIVEIRA et al., 2012).

Este estudo de revisão tem como objetivo compreender os aspectos fisiológicos e terapêuticos da incontinência urinária e sua influência na qualidade de vida do incontinente, apresentando propostas de como a enfermagem pode atuar para o bem-estar físico, emocional e social dos portadores. Para tal, foram utilizadas como fonte de pesquisa as bases de dados científicos eletrônicos PubMed, SciELO, portal de periódicos CAPES, EBSCO e DynaMed e selecionados 32 artigos com recorte temporal de 2002 a 2018.

\section{RESULTADO E DISCUSSÃO}

\section{Fisiopatologia, fatores de risco e epidemiologia da IU}

A ação de armazenar e esvaziar a bexiga é um ciclo fisiológico complexo, cuja consequência básica é a micção. Para que esse ciclo ocorra é necessário que a estrutura anatômica, com diferentes músculos e os nervos parassimpáticos, simpáticos, somáticos e sensoriais, trabalhem em conjunto. Qualquer alteração estrutural ou funcional na bexiga, uretra e/ou esfíncteres, principalmente ao acontecer o esvaziamento, pode resultar no desenvolvimento de incontinência (CÂNDIDO et al., 2017; OLIVEIRA et al., 2012).

A IU pode ser classificada em diferentes tipos clínicos, de acordo com a sintomatologia e o mecanismo fisiopatológico, assim sendo mencionada como transitória ou crônica. A transitória é a perda urinária momentânea que se reverte espontaneamente, após tratar o que a está causando. Para isso, é necessário estar presente há menos de seis semanas, sendo suas principais causas: momento de confusão aguda, infecção sintomática do trato urinário, uretrite atrófica, vaginite, medicamentos, deficiências psicomotoras e compactação das fezes. Espera-se que o paciente recupere a continência quando essas causas são devidamente solucionadas (CÂNDIDO et al., 2017; KHANDELWAL e KISTLER, 2013; BLANCHETTE, 2012; OLIVEIRA et al., 2012).

A IU crônica geralmente não se reverte espontaneamente e pode ser dividida em alguns tipos como: incontinência de esforço, incontinência de urgência, incontinência mista, incontinência de transbordamento ou paradoxal, incontinência funcional e a incontinência contínua (KHANDELWAL e KISTLER, 2013).

A perda involuntária de urina durante uma ação de esforço, caracteriza-se como a IU de esforço. Esse escape acontece ao realizar atividades como tossir, rir, espirrar, subir ou descer escadas, correr e levantar, devido a um aumento da pressão intravesical. Apresenta como causa a fraqueza do esfíncter ou a fraqueza do assoalho pélvico, que consequentemente, irá gerar uma função ineficaz do sistema urinário. É o tipo mais comum entre as mulheres jovens, representando até $65 \%$ de todos os tipos de IU em mulheres, e o segundo em idosas, podendo também ocorrer em homens após cirurgia de próstata (CÂNDIDO et al., 2017; AGARWAL e AGARWAL, 2017; OPARA e CZERWIŃSKA-OPARA, 2014; KHANDELWAL e KISTLER, 2013). 
A necessidade repentina, forte e imperiosa de urinar, ocorrendo à perda de urina antes de conseguir chegar ao banheiro, caracteriza-se como a IU de urgência. Essa é decorrente da hiperatividade do músculo detrusor, que pode apresentar causa sensorial, decorrente de uma irritação, inflamação ou infecção no interior da bexiga, e/ou distúrbios neurológicos sensitivos (CÂNDIDO et al., 2017; KHANDELWAL e KISTLER, 2013; BORBA et al., 2008).

A presença simultânea dos mecanismos fisiopatológicos da IU de esforço e de urgência resulta na IU mista (LEÓN et al., 2017; CÂNDIDO et al., 2017).

A IU paradoxal ou por transbordamento, é sequente de uma retenção urinária, causada pelo comprometimento da contratilidade do detrusor, ou por uma obstrução da saída da bexiga, ou até mesmo por ambas as causas juntas. Como consequência, terá uma distensão da bexiga, que levará a um escape de urina por estouro, ou seja, quando a bexiga está completamente cheia, a urina transborda involuntariamente. O paciente apresenta a vontade de urinar, mas elimina apenas gotas. Esse tipo é mais comum em homens de forma crônica, devido à hiperplasia prostática (CÂNDIDO et al., 2017; KHANDELWAL e KISTLER, 2013).

As dificuldades cognitivas, funcionais ou de mobilidade que prejudica o uso do banheiro, e que não há falha funcional da bexiga ou no controle neurológico, caracteriza-se como a IU funcional. Ou seja, é a perda involuntária de urina, causada por barreiras ambientais ou físicas para o acesso ao banheiro (KHANDELWAL e KISTLER, 2013).

Por último a IU contínua caracteriza-se como a perda constante de urina, causada por lesões graves ao sistema esfincteriano, no qual a pressão uretral torna-se incapaz de impedir o fluxo urinário. Essa pode ser congênita ou consequente de ressecções pélvicas e traumas genitais (CÂNDIDO et al., 2017).

A incontinência pode ser causada por múltiplos fatores, dentre os mais prevalentes encontram-se: envelhecimento, genética, raça branca, obesidade, poli farmácia, tabagismo, constipação, alimentos irritantes vesicais, doenças crônicas, infecções do trato urinário, cirurgias pélvicas, atividades físicas de alto impacto na região abdominal, trauma do assoalho pélvico, enfermidades neurológicas, déficit cognitivo e funcional. Além desses, as mulheres possuem alguns fatores específicos como: gravidez, histórico obstetrício, histerectomia, menopausa, terapia hormonal substitutiva e cirurgia ginecológica (CÂNDIDO et al., 2017; TOMASI et al., 2017; BENÍCIO et al., 2017; JUNQUEIRA e SANTOS, 2017; LEÓN et al., 2017; OPARA e CZERWIŃSKA-OPARA, 2014; KHANDELWAL e KISTLER, 2013; BLANCHETTE, 2012; OLIVEIRA et al., 2012).

Considerada como uma questão prioritária de saúde, a IU é subnotificada, subdiagnosticada e apresenta baixo reconhecimento profissional. Essa patologia pode ser um sintoma presente em várias doenças, afeta a todos os grupos populacionais, não importando idade, sexo ou etnia. Mesmo ocorrendo mais em mulheres, depois de certa faixa etária apresenta uma incidência semelhante entre ambos os sexos, portanto sua prevalência cresce de acordo com o aumento da idade populacional. Acomete cerca de 400 milhões de pessoas no mundo, ou seja, aproximadamente a cada 200 pessoas 1 é incontinente, e conforme a Sociedade Brasileira de Urologia (SBU), no Brasil há em média 10 milhões de incontinentes (LEÓN et al., 2017; AGARWAL e AGARWAL, 2017; RIEMSMA et al., 2017; BENÍCIO et al., 2017; JUNQUEIRA e SANTOS, 2017; HUTCHINGS e SUTHERLAND, 2014).

\section{Como a IU Influência na vida do incontinente}

A IU não é apenas um problema fisiológico, mas também psicológico, que traz consequências para o social, o econômico, o ocupacional, o doméstico e o sexual de uma pessoa, ou seja, afeta diretamente a qualidade de vida. Essa patologia pode repercutir como um trauma psicológico, uma ameaça para a 
autoestima, depressão, isolamento, ansiedade, dificuldades relacionadas ao trabalho, perda da independência, vulnerabilidade e sentimentos como: solidão, culpa, desespero, impotência, angustia e humilhação. Para os idosos além de todos os impactos de vida, a incontinência ainda aumenta a probabilidade de institucionalização em lares de longa permanência (LEÓN et al., 2017; LUO et al., 2016; OPARA e CZERWIŃSKA-OPARA, 2014; HUTCHINGS e SUTHERLAND, 2014; KHANDELWAL e KISTLER, 2013; KRISTIANSEN, 2011; HONÓRIO e SANTOS, 2009; BORBA et al., 2008).

O escape involuntário de urina torna-se então algo constrangedor, ocasionando a restrição social, pois o medo do odor transparecer impede que muitas pessoas frequentem lugares de convívio comum. Essa questão está relacionada com as crenças e ideias culturais do ser humano, que associa a perda do controle urinário com o uso de fralda e falta de higiene (CÂNDIDO et al., 2017; HIGA et al., 2010).

Para conviver com a incontinência, as pessoas passam a ter modificações comportamentais a fim de minimizar as inconveniências. Muitas alteram seus hábitos diários passando a utilizar perfumes de odor forte, roupas escuras, absorventes ou protetores para controle da perda da urina. Mudam também a ingestão hídrica, tomando menos líquido ao decorrer do dia, trocam inúmeras vezes de roupas, ficam sempre próximas a banheiros, suspendem fármacos que estimulam a função do sistema urinário por conta própria, e evitam o convívio social (KRISTIANSEN, 2011; HIGA et al., 2010; BORBA et al., 2008).

\section{Avaliação da IU}

Grande parte dos incontinentes não procuram uma ajuda profissional, seja devido a barreiras culturais, físicas, sociais ou até mesmo psicológicas. Muitas das vezes, essas pessoas ou até mesmo seus cuidadores acham a perda involuntária de urina como algo cômodo, normal e sem importância, devido ao envelhecimento, inexistindo a necessidade de procurar um serviço de saúde (FRANKEN et al., 2018; CÂNDIDO et al., 2017; TOMASI et al., 2017; TALLEY et al., 2017; LUO et al., 2016; HUTCHINGS e SUTHERLAND, 2014; OLIVEIRA et al., 2012; BARBOSA et al., 2009).

O constrangimento que muitas pessoas têm ao falar a respeito dessa patologia com os familiares, amigos ou até mesmo com um profissional de saúde, também se torna uma barreira para procurar uma solução clínica. Entretanto, a procura precoce de uma avaliação diagnóstica e uma orientação profissional, resultará uma resposta terapêutica mais satisfatória (LEÓN et al., 2017; TOMASI et al., 2017; HUTCHINGS e SUTHERLAND, 2014; OLIVEIRA et al., 2012).

A avaliação da IU deve apresentar como objetivo a identificação de fatores reversíveis e de determinação estratégica apropriada para minimizar e solucionar o escape de urina. Essa é realizada por meio de uma anamnese, de um exame físico e de uma avaliação urodinâmica. A anamnese deve apresentar uma avaliação geral e uma específica do paciente. Na específica é importante conter dados como: início dos sintomas, duração, impactos, associação com outras comorbidades, função sexual e intestinal, história ginecológica, história urológica pregressa, histórico neurológico, histórico cirúrgico e registro do diário miccional (KHANDELWAL e KISTLER, 2013; ALBA et al., 2011; HANZAREE e STEGGALL et al., 2010 FELDNER JR et al., 2002).

Durante o relato da história clínica, é importante obter a percepção do problema pelo paciente e o impacto em sua qualidade de vida. Para isso, pode ser utilizado questionários a respeito da qualidade de vida como por exemplo: EuroQol EQ-5D, I-QoL, King's Health Questionnaire (KHQ), I-PSS, ICIQ-SF. Estes são ferramentas úteis que vão verificar os sintomas presentes e seus respectivos impactos na vida diária (CÂNDIDO et al., 2017; SABOIA et al., 2017; ALBA et al., 2011; FELDNER JR et al., 2002).

Quanto ao exame físico, deve ser realizado céfalo-podálico, porém com ênfase nas regiões abdominais e pélvicas. Para uma melhor análise dos sinais de incontinência no exame, indica-se explorar o paciente 
com bexiga de reabastecimento moderado, verificar sinais de IU de esforço, e realizar manobras de valsava. Caso não for possível perceber os sinais com o paciente em decúbito, o mesmo deve ser examinado em pé com as pernas entre abertas (ALBA et al., 2011).

A última avaliação a ser realizada é a urodinâmica, com estudos e exames específicos do sistema urinário, identificando as causas singulares e confirmando um diagnóstico final. Após todos os dados coletados e estudados nas avaliações, é possível orientar o tratamento mais indicado (FELDNER JR et al., 2002).

A avaliação da IU deve ser integral, completa, e realizada por uma equipe multiprofissional, para uma intervenção necessária e eficaz, pois um diagnóstico incorreto pode resultar múltiplas consequências, como a indicação de cirurgias em situação inapropriada ou desnecessária (BARBOSA et al., 2009; FELDNER JR et al., 2002).

\section{Tratamento da IU}

No tratamento da IU existem muitas opções, que podem ser realizadas em conjunto, como: as terapias comportamentais, exercícios da musculatura pélvica (EMP), terapia com cones vaginais, eletroestimulação transvaginal ou retal, medicações, cirurgias e produtos de contenção (absorventes, fraldas e cateteres bolsa coletora de urina). O que vai determinar a escolha do tratamento é a gravidade dos sintomas e a influência na qualidade de vida do incontinente (RIEMSMA et al., 2017; BENícIO et al., 2017; LOPES et al., 2016; KHANDELWAL e KISTLER, 2013; OLIVEIRA et al., 2012).

A IU, além de gerar muitos efeitos físicos e psicológicos, é associada também a riscos de lesões e problemas de pele, que faz estender o tratamento do incontinente (FRANKEN et al., 2018).

Em grande parte, os profissionais de saúde escolhem uma alternativa não curativa, mas que ajudam a mascarar os problemas, prescrevendo o uso de absorventes ou fraldas para incontinentes, ou seja, indicam a terapia de contenção. Essa é utilizada por mais de $50 \%$ dos portadores, principalmente por idosos, mesmo considerada de alto custo (AGARWAL e AGARWAL, 2017; ALBERS-HEITNER et al., 2011).

O tratamento não invasivo é a opção inicial mais adequada de intervenção, que vai buscar a diminuição da sintomatologia e a solução do problema. Com isso, a primeira opção são as terapias comportamentais, de baixo risco e custo, buscam modificações comportamentais e mudanças em hábitos diários de vida. $\mathrm{A}$ terapia comportamental, podendo ser da competência do enfermeiro, incluindo estratégias como: controle hídrico, alteração da alimentação, adaptação miccional de acordo com os episódios de incontinência, reeducação da bexiga e exercício da musculatura pélvica (EMP). Na alimentação devem-se evitar consumos de alimentos considerados irritantes vesicais, como cafeínados, frutas ácidas, achocolatados e refrigerantes, ou seja, produtos que podem agravar episódios de perdas urinárias, principalmente na IU de urgência (VALENÇA et al., 2016; OLIVEIRA et al., 2012; BLANCHETTE, 2012; ALBA et al., 2011; ALBERSHEITNER et al., 2011; HANZAREE e STEGGALL et al., 2010; HONÓRIO e SANTOS, 2009).

Também conhecidos como exercícios de Kegel, os EMP têm como objetivo fortalecer e reabilitar o assoalho pélvico, buscando melhorar a eficiência esfincteriana e impedir a perda de urina. EMP é a contração e o relaxamento da musculatura pélvica, e deve ser realizado 02 ou 03 vezes por semana. Esse exercício deve ser monitorado através da técnica de biofeedback, que vai verificar a contração muscular através de eletromiografia, permitindo visualizar a ação muscular (CÂNDIDO et al., 2017; LOPES et al., 2016; SANGSAWANG; SERISATHIEN, 2012; OLIVEIRA et al., 2012; CALDAS et al.,2010).

Além do EMP, existe também a terapia com cones vaginais e a eletroestimulação transvaginal ou retal. A terapia com cones é realizada com cones de peso de 20 a 100 gramas que serão introduzidos na vagina, 
para ficarem retidos através da contração muscular por um período de tempo. Já a eletroestimulação é um exercício artificial, que aumenta a contratilidade dos músculos pélvicos e o tônus muscular. Esse é realizado através de eletrodos que são colocados na vagina ou no reto para estimulação elétrica ou magnética, sendo mais eficaz quando o distúrbio é funcional e não anatômico (CÂNDIDO et al., 2017; LOPES et al., 2016; OLIVEIRA et al., 2012).

A última escolha para tratamento é o cirúrgico, no qual é realizado apenas, quando todos os outros métodos não foram eficazes. Geralmente nesses casos tem-se uma reconstituição de elementos, como utilização de slings e colpofixação retropúbica, para IU de esforço e hipermobilidade da uretra (CÂNDIDO et al., 2017; OLIVEIRA et al., 2012).

O cuidado com a IU deve ser prestado de forma contínua considerando o paciente como um todo, e para isso deve ultrapassar as barreiras de relacionamento que existem entre paciente, profissional e o serviço de saúde, evitando uma assistência mecanizada. Com isso, a motivação do autocuidado e a passagem de informação sobre a patologia são aspectos importantes, para que os pacientes entendam melhor seus problemas e facilitam as terapias, proporcionando uma satisfação na busca de seu tratamento (CÂNDIDO et al., 2017; TOMASI et al., 2017; HUTCHINGS e SUTHERLAND, 2014; ALBERS-HEITNER et al., 2011; HONÓRIO e SANTOS, 2009).

\section{Atuação da enfermagem}

Os enfermeiros são profissionais que podem prestar assistência aos incontinentes, sendo capazes de avaliar, identificar, fornecer informações e estabelecer algumas intervenções adequadas em diversos contextos, seja em paciente inserido na comunidade ou em hospitais, apresentando cuidados agudos ou até mesmo pós-agudos (HUTCHINGS e SUTHERLAND, 2014; ALBERS-HEITNER et al., 2011).

A enfermagem possui uma área de especialidade para avaliar e manusear a IU, denominada como estomaterapia que compreende assistência às pessoas que apresentam estomas, feridas, incontinência anal e urinária. Essa especialidade é reconhecida pelo órgão de classe e sociedades cientificas nacionais e internacionais, porém existem poucos enfermeiros especializados e inseridos nessa área (SILVA e D'ELBOUX, 2012).

A assistência de enfermagem vai além de cuidados domiciliares focados apenas em utilização de absorventes para incontinência, deve ser desempenhada uma assistência integral ao incontinente que irá contribuir para o controle da perda urinaria e melhora na qualidade de vida dos pacientes. Uma anamnese, um exame físico completo e uma atenção sistematizada bem executados pelo enfermeiro podem resultar na identificação da IU, trazendo um diagnóstico preciso e intervenções necessárias (VALENÇA et al., 2016; ALBERS-HEITNER et al., 2010).

O enfermeiro pode atuar com o incontinente, através da educação em saúde, do suporte emocional, da terapia comportamental e da Sistematização da Assistência de Enfermagem (SAE). Esse almeja não só uma condição terapêutica física, mas também uma melhora integral do paciente, abrangendo o lado psicossocial e consequentemente possibilitando uma melhor qualidade de vida (VALENÇA et al., 2016).

A educação em saúde auxilia o paciente no processo de percepção, enfretamento, reabilitação, adaptação e aceitação ao tratamento, buscando a orientação e o autocuidado como a melhor opção de enfrentar a IU. O enfermeiro pode realizar todo esse processo através da explicação do mecanismo fisiopatológico e do que é ser incontinente, esclarecendo dúvidas e mitos. É importante também retratar a respeito da associação e dissociação da IU com o envelhecimento, como um processo natural da vida; e trabalhar a prevenção de acidentes físicos, como quedas de pacientes idosos, relacionadas ao aumento da frequência de micção (VALENÇA et al., 2016; HUTCHINGS e SUTHERLAND, 2014). 
No suporte emocional, o enfermeiro pode estimular o incontinente a participar de grupos de apoio que permitem a troca de experiência. Pode também proporcionar confiança e empatia na relação de enfermeiro/paciente, incentivar ao retorno das atividades diárias sem colocar limitações e ampliar a compreensão do paciente a respeito da patologia e suas consequências. É importante a avaliação precisa da qualidade de vida desses, para identificar estratégias efetivas de abordagem e buscar minimizar o sofrimento psicológico, incentivando a promoção da saúde e o autocuidado (VALENÇA et al., 2016; HUTCHINGS e SUTHERLAND, 2014).

$\mathrm{Na}$ terapia comportamental o enfermeiro pode auxiliar o incontinente na mudança do estilo de vida, nos cuidados de higiene e na prevenção de infecção; estimular a prática de exercício físico e a redução de peso; orientar quanto à ingesta hídrica e ao consumo de alimentos não constipantes e não irritantes vesical; influenciar no processo de fortalecimento da musculatura do assoalho pélvico com exercícios; e outros processos comportamentais que promovam a melhora e diminuição da perda involuntária da urina (VALENÇA et al., 2016; OLIVEIRA et al., 2012).

$\mathrm{Na}$ SAE, o enfermeiro pode realizar a investigação precoce dos sintomas de IU através da anamnese e do exame físico durante a consulta de enfermagem, e posteriormente produzir um planejamento dos cuidados a serem executados pela sua equipe e paciente. Para a elaboração de cuidados, deve ser levado em consideração o que foi decidido acerca das questões do tratamento, ser verificado e quantificado os riscos de lesões e cuidados com a pele, compreendendo a rede de apoio familiar, as relações sociais e as possíveis modificações para melhorar a qualidade de vida do paciente. Vale ressaltar a necessidade de uma assistência ao incontinente, baseada nos processos de enfermagem, pois essa considera o paciente em sua totalidade (VALENÇA et al., 2016).

A falta de informação e de conhecimentos dos profissionais de enfermagem, cria uma barreira para as intervenções adequadas relacionadas à IU, e para ser quebrada é necessário o conhecimento prático, compreensão básica das causas, técnicas de avaliação, técnicas de diagnóstico, técnicas de manejo e métodos de intervenção por parte desses profissionais (HUTCHINGS e SUTHERLAND, 2014; BLANCHETTE, 2012).

\section{CONSIDERAÇÕES FINAIS}

A perda involuntária de urina é um incômodo na vida de uma pessoa e influencia diretamente sua rotina diária. Devido a escassos estudos sobre incontinência e falta de informação a respeito da fisiopatologia por parte dos portadores e dos profissionais de saúde, a IU passa a ser considerada como algo natural e secundário. Poucos profissionais de enfermagem conhecem ou investigam a respeito desse tema, que necessita ser mais explorado e difundido, para realizar uma assistência adequada dos incontinentes. Os enfermeiros podem desempenhar um papel importante na identificação dos sintomas e na interação com o paciente, incentivando as mudanças comportamentais e o tratamento clínico. Atuando então diretamente para o bem-estar físico, emocional e social dos portadores dessa patologia, e melhorando sobretudo a qualidade de vida.

\section{REFERÊNCIAS}

1. ABRAMS P, CARDOZO L, FALL $M$ et al. The standardisation of terminology in lower urinary tract function: report from the standardisation sub-committee of the international continence society. Elsevier Science, 2003; 61: 37-49.

2. AGARWAL BK, AGARWAL N. Urinary incontinence: prevalence, risk factors, impact on quality of life and treatment seeking behaviour among middle aged women. International Surgery Journal, 2017; 4(6): 1953-1958.

3. ALBA DV, ALARMA SB, REINA GR et al. Incontinencia urinaria. Medicine, 2011;10(83):5612-8. 
4. ALBERS-HEITNER CP, LAGRO-JANSSEN ALM, VENEMA PL et al. Experiences and atitudes of nurse specialists in primary care regarding their role in care for patients with urinary incontinence. Scand J Caring Sci, 2011; 25: 303310.

5. BARBOSA SS, OLIVEIRA LDR, LIMA JLDA et al. Como profissionais de saúde da rede básica identificam e tratam a incontinência urinária feminina. O Mundo da Saúde, São Paulo: 2009;33(4): 449-456.

6. BENÍCIO CDAV, LUZ MHBA, CARVALHO NV et al. Conhecimento de mulheres incontinentes sobre incontinência urinária: uma reflexão teórico-crítica. Estima, 2017; 15(1): 58-61.

7. BLANCHETTE KA. (2012). Exploration of nursing care strategies for the management of urinary incontinence in hospitalized women. Urologic Nursing, 2012; 32(5), 256-271.

8. BORBA AMC, LELIS MAS, BRÊTAS ACP. Significado de ter incontinência urinária e ser incontinente na visão das mulheres. Texto Contexto Enferm, Florianópolis, 2008; 17(3): 527-535.

9. CALDAS CP, CONCEIÇÃO IRS, JOSÉ RMC et al. Terapia comportamental para incontinência urinária da mulher idosa: uma ação do enfermeiro. Texto \& Contexto Enfermagem, 2010; 19(4): 783-788.

10. CÂNDIDO FJLF, MATNEI T, GALVÃO LC et al. Incontinência urinária em mulherer: breve revisão de fisiopatologia, avaliação e tratamento. Visão Acadêmica, Curitiba, 2017; 18(3): 67-80.

11. FELDNER JR PC, BEZERRA LRPS, GIRÃO MJBC et al. Valor da Queixa Clínica e Exame Físico no Diagnóstico da Incontinência Urinária. RBGO, 2002; 24(2): 87-91.

12. FRANKEN MG, RAMOS IC, LOS $\mathrm{J}$ et al. The increasing importance of a continence nurse specialist to improve outcomes and save costs of urinary incontinence care: na analysis of future policy scenarios. BMC Family Practice, 2018; 31(19): 1-11.

13. HIGA R, RIVORÊDO CRSF, CAMPOS LK et al. Vivências de mulheres brasileiras com incontinência urinária. Texto \& Contexto Enfermagem, 2010; 19(4): 627-635.

14. HONÓRIO MO, SANTOS SMA. Incontinência urinária e envelhecimento: impacto no cotidiano e na qualidade de vida. Revista Brasileira de Enfermagem, 2009; 62(1): 51-56.

15. HANZAREE Z, STEGGALL MJ. Treatment of patients with urge or stress urinary incontinence. Nursing Standard, 2010; 25(3): 41-46.

16. HUTCHINGS J, SUTHERLAND, L. Student nurse understanding of the psychosocial impact of urinary incontinence. Urologic Nursing, 2014; 34(6), 318-325.

17. JUNQUEIRA JB, SANTOS VLCG. Incontinência urinária em pacientes hospitalizados: prevalência e fatores associados. Rev. Latino-Am. Enfermagem, 2017;25:e2970.

18. KHANDELWAL C, KISTLER C. Diagnosis of urinary incontinence. American Family Physician, 2013; 87(8): 543-550.

19. KRISTIANSEN L, BJORK A, KOCK VB et al. Urinary incontinence ande newly invented pad technique: patients', close relatives' and nursing staff's experiences and beliefs. Internacional Journal of Urological Nursing, 2011; 5(1): 21-30.

20. LEÓN CG, PÉREZ-HARO ML, JALÓN-MONZÓN A et al. Actualización en incontinencia urinaria feminina. Semergen. 2017; 43(8): 578-584.

21. LOPES MHBM, COSTA JN, LIMA JLDAL et al. Programa de reabilitação do assoalho pélvico: relato de 10 anos de experiência. Revista Brasileira de Enfermagem, 2017; 70(1): 219-223.

22. LUO Y, PARRY M, HUANG Y et al. Nursing students' knowledge and atitudes toward urinary incontinence: A crosssectional survey. Nurse Education Today, 2016; 40: 134-139.

23. MENDES KDS, SILVEIRA RCCP, GALVÃO CM. Revisão integrativa: método de pesquisa para a incorporação de evidências na saúde e na enfermagem. Texto Contexto Enfermagem, 2008; 17(4): 758-764.

24. OLIVEIRA AM, CAMARGO ACS, SCHELL NC et al. Assistência de enfermagem a incontinência urinária na mulher. Múltiplo saber, 2012; 15(1):100-111.

25. OPARA J, CZERWIŃSKA-OPARA WE. The prevalence of stress urinary incontinence in women studying nursing and related quality of life. Prz Menopauzalny 2014; 13(1): 32-35.

26. RIEMSMA R, HAGEN S, KIRSCHNER-HERMANNS R et al. Can incontinence be cured? A systematic review of cure rates. BMC Medicine, 2017; 63(15): 1-11.

27. SABOIA DM, FIRMIANO MLV, BEZERRA KC et al. Impact of urinary incontinence types on women's quality of life. Rev Esc Enferm USP. 2017;51:e03266.

28. SANGSAWANG B, SERISATHIEN Y. Effect of pelvic floor muscle exercise programme on stress urinary incontinence among pregnant women. Journal of Advanced Nursing, 2012; 68(9): 1997-2007.

29. SILVA VA, D'ELBOUX. Atuação do enfermeiro no manejo da incontinência urinária no idoso: uma revisão integrativa. Rev Esc Enferm USP, 2012; 46(5): 1221-1226.

30. TALLEY KMC, DAVIS NJ, WYMAN JF. Determining a treatment plan for urinary incontinence in an older adult: Application of the four-topic approach to ethical decision-making. Urologic Nursing, 2017; 37(4): 181-191.

31. TOMASI AVR, SANTOS SMA, HONÓRIO JS et al. Urinary incontinence in elderly people: care practices and care proposal in primary health care. Texto Contexto Enfermagem, 2017; 26(2): e6800015.

32. VALENÇA MP, ALBUQUERQUE AFLL, ROCHA GMSR et al. Cuidados de Enfermagem na Incontinência Urinária: um Estudo de Revisão Integrativa. ESTIMA, 2016; 14(1): 43-49. 33. 